Southampton 


\section{Ontology Design Patterns}

\section{COMP6215 Semantic Web Technologies}

Dr Nicholas Gibbins - nmg@ecs.soton.ac.uk 


\section{Design Patterns}

Patterns are general, reusable solutions to commonly occurring problems

- Concept originated with Christopher Alexander's work on architecture

- Popularised in software engineering by the "gang of four"

- Subject of study by the knowledge engineering community

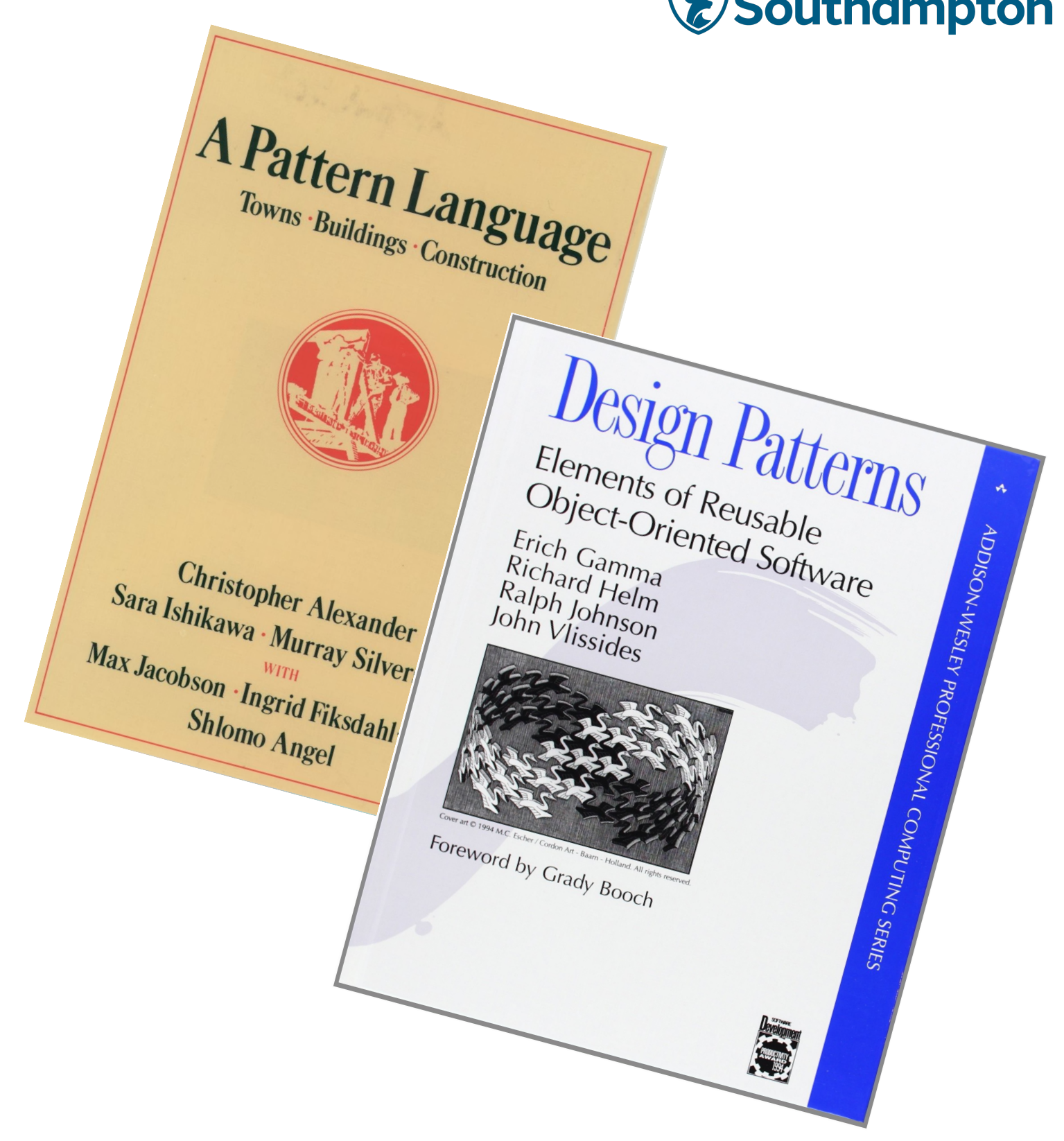




\section{Design Patterns for the Semantic Web}

$\mathrm{N}$-ary relations

- How can we say more about a relation instance?

- How do we represent an ordered sequence of relations?

Value partitions and value sets

- How do we represent a fixed list of values?

Part-whole hierarchies

- How do we represent hierarchies other than the subclass hierarchy? 
University of

Southampton

\section{$\mathrm{N}$-ary Relations}




\section{Binary Relations}

In RDF and OWL, binary relations link two individuals, or an individual and a value

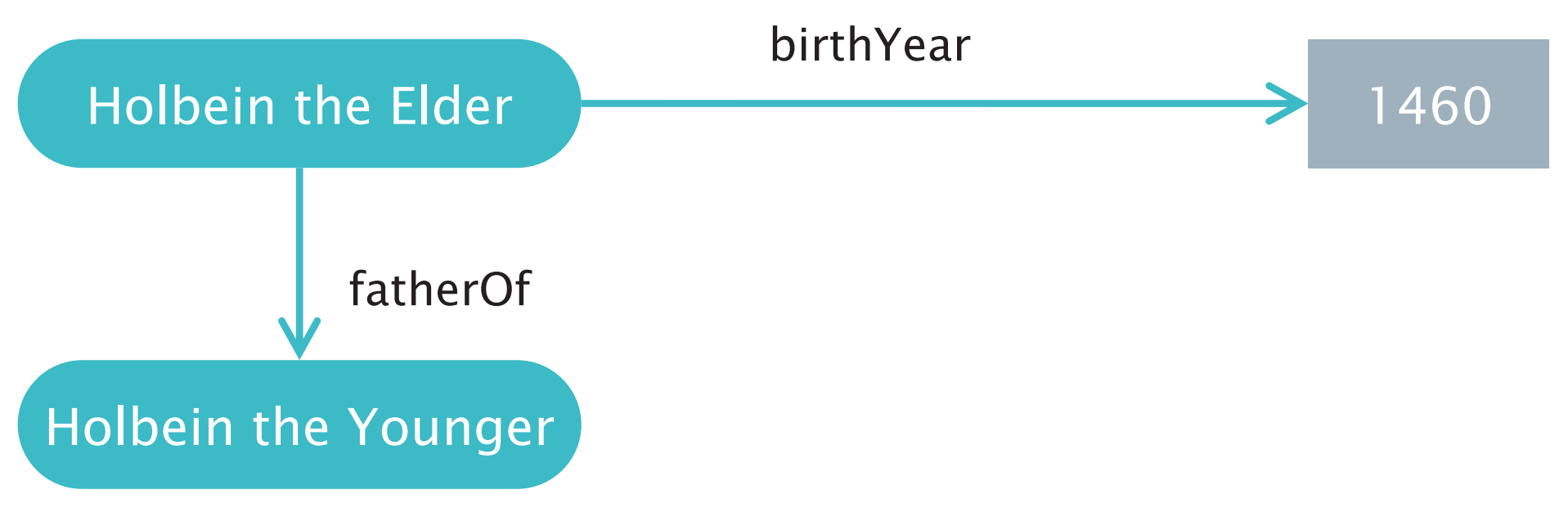

The properties birthYear and fatherOf are binary relations 


\section{Relations with Additional Information}

In some cases, we need to associate additional info with a binary relation

- e.g. certainty, strength, dates

For example, Holbein the Elder's date of birth is unconfirmed

- He was born in either 1460 or 1465

- How can we represent this uncertainty?

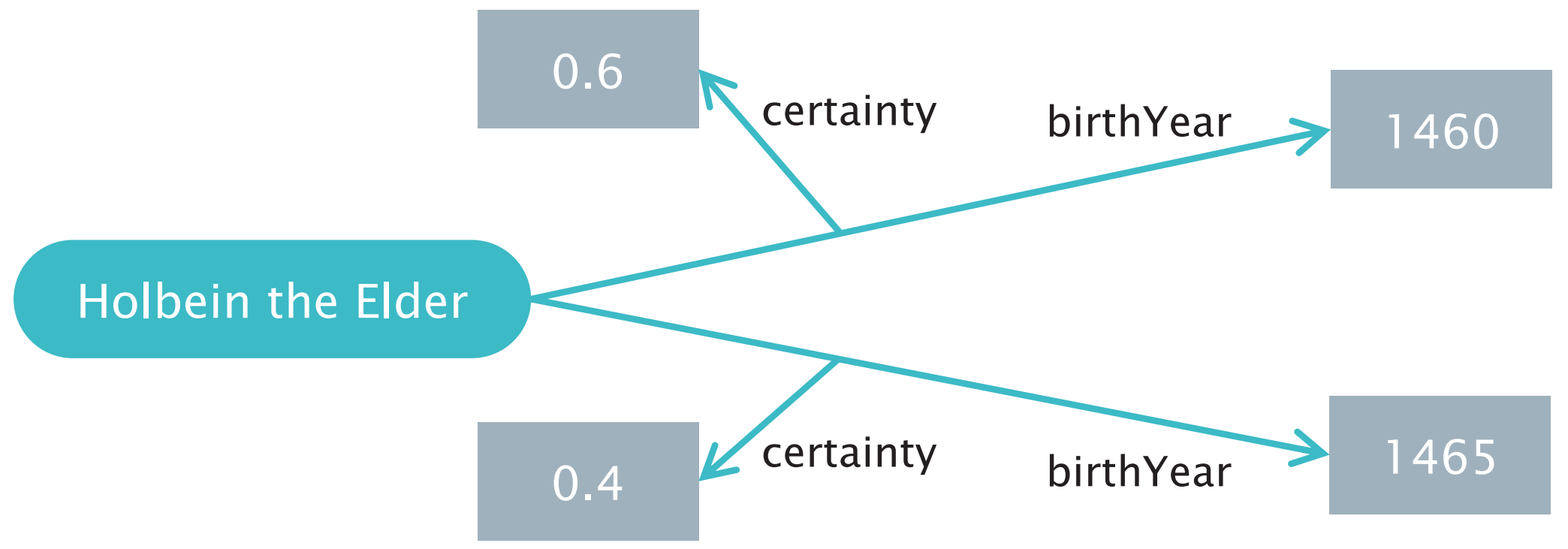




\section{$\mathrm{N}$-ary Relations}

$\mathrm{N}$-ary relations link an individual to more than a one value

Possible use cases:

1. A relation needs additional info e.g. a relation with a rating value

2. Two binary relations are related to each other e.g. body_temp (high, normal, low), and trend (rising, falling)

3. A relation between several individuals e.g. someone buys a book from a bookstore

4. Linking from, or to, an ordered list of individuals e.g. an airline flight visiting a sequence of airports 


\section{Pattern 1: Reified Relation}

To represent additional information about a relation:

- Create a new class to represent the relation

- Individuals of this class are instances of the relation

- Relation class can have additional properties to describe more information about the relation

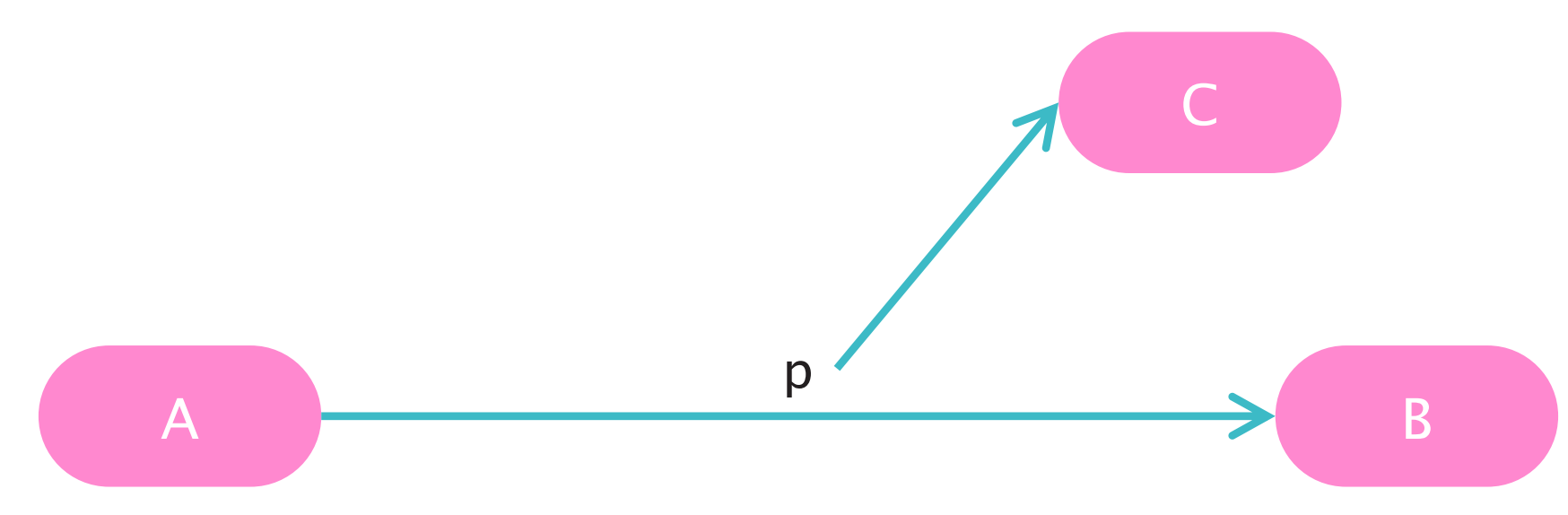




\section{Use case 1: additional information}

Jack has given the film 'I Am Legend' a four-star rating

-We need to represent a quantitative value to describe the rating relation

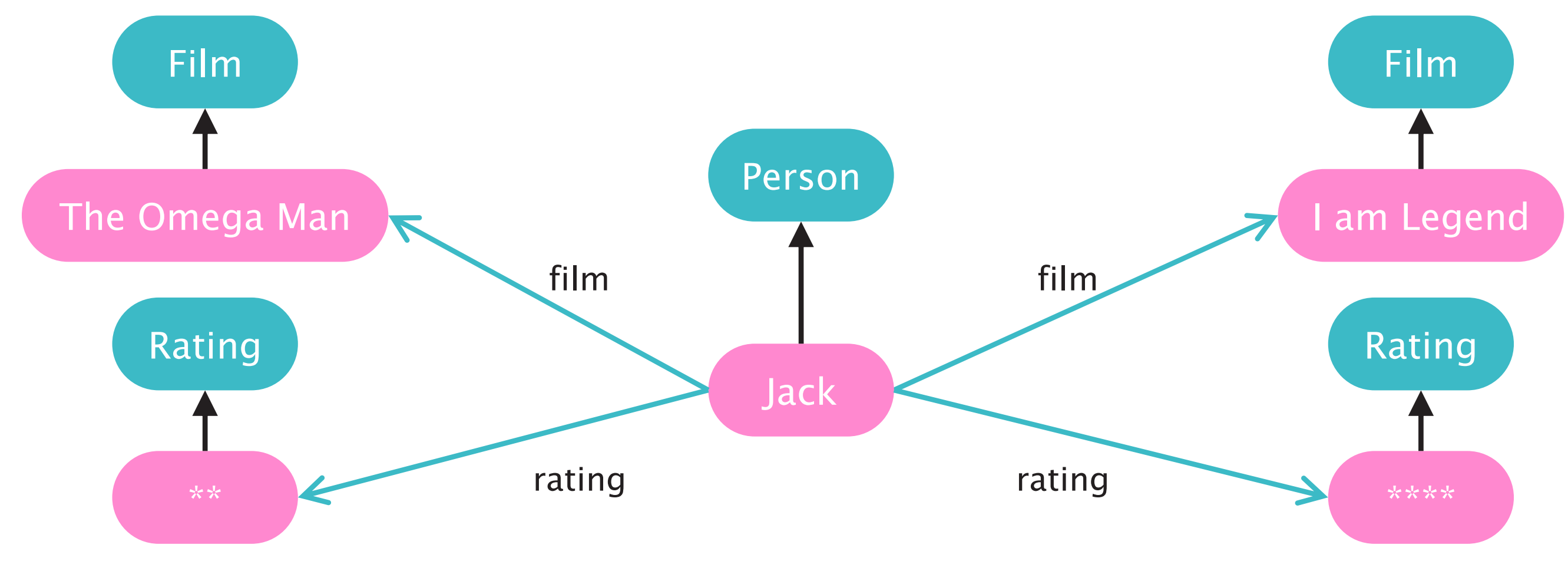




\section{Use case 1: additional information}

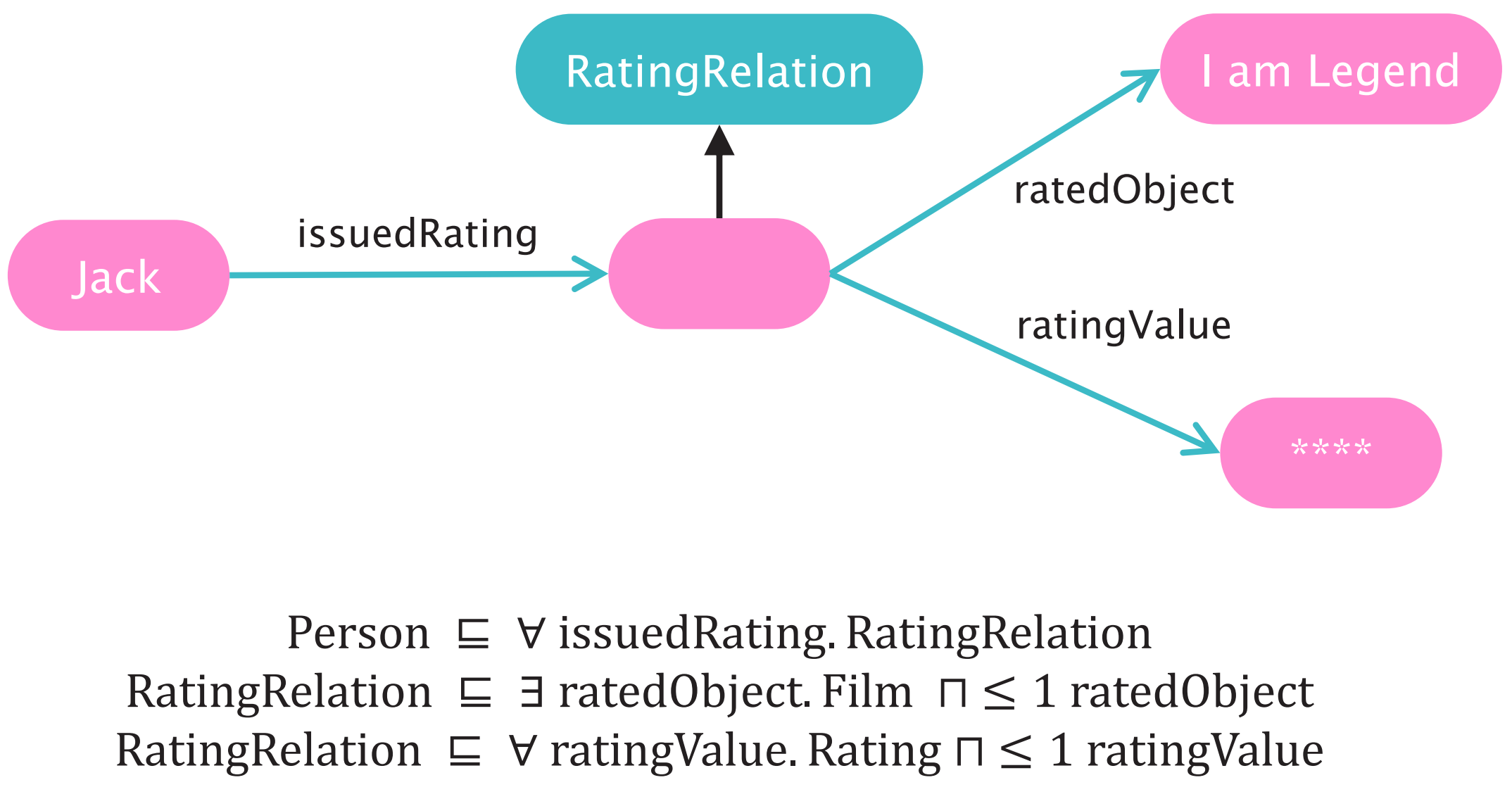




\section{Use case 2: different aspects of a relation}

Steve has a temperature which is high, but falling

-We need to represent different aspects of the temperature that Steve has

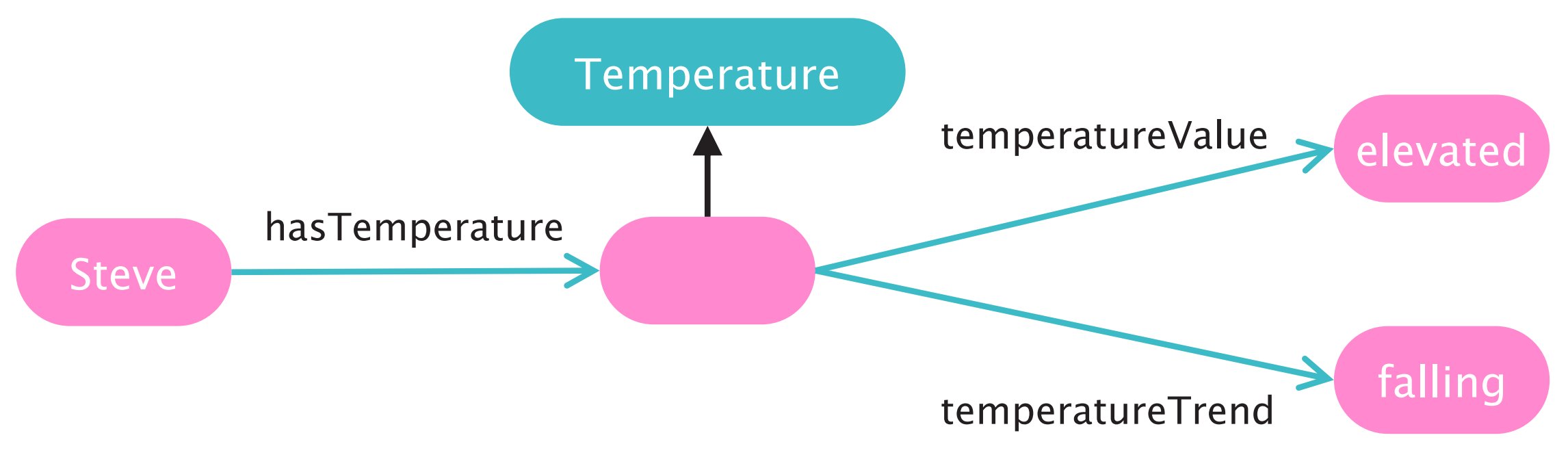




\section{Use case 3: no distinguished participant}

John buys a "Lenny the Lion" book from orinoco.com for $\$ 15$ as a birthday gift

- No distinguished subject for the relation

- i.e. no primary relation to convert into a Relation Class as in cases 1 and 2

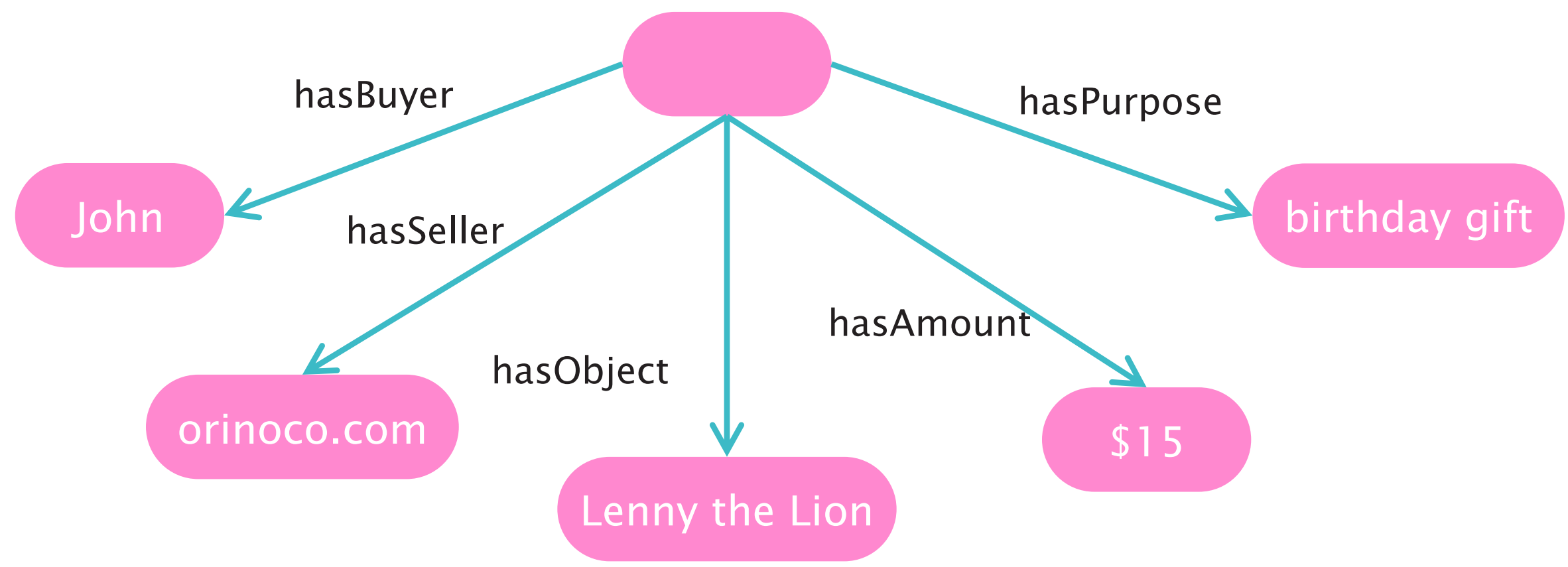




\section{Use case 3: no distinguished participant}

Purchase $ᄃ \exists$ hasBuyer. Person $\Pi=1$ hasBuyer

Purchase $\subseteq \exists$ hasSeller. Company $\Pi=1$ hasSeller

Purchase $\subseteq \exists$ hasObject. Object

Purchase $\subseteq \forall$ hasAmount. Quantity $\Pi=1$ hasAmount

Purchase $\subseteq \forall$ hasPurpose. Purpose 


\section{Pattern 2: Sequence of arguments}

United Airlines, flight 1377 visits the following airports: LAX, DFW, and JFK

- For such an example, we need to represent a sequence of arguments

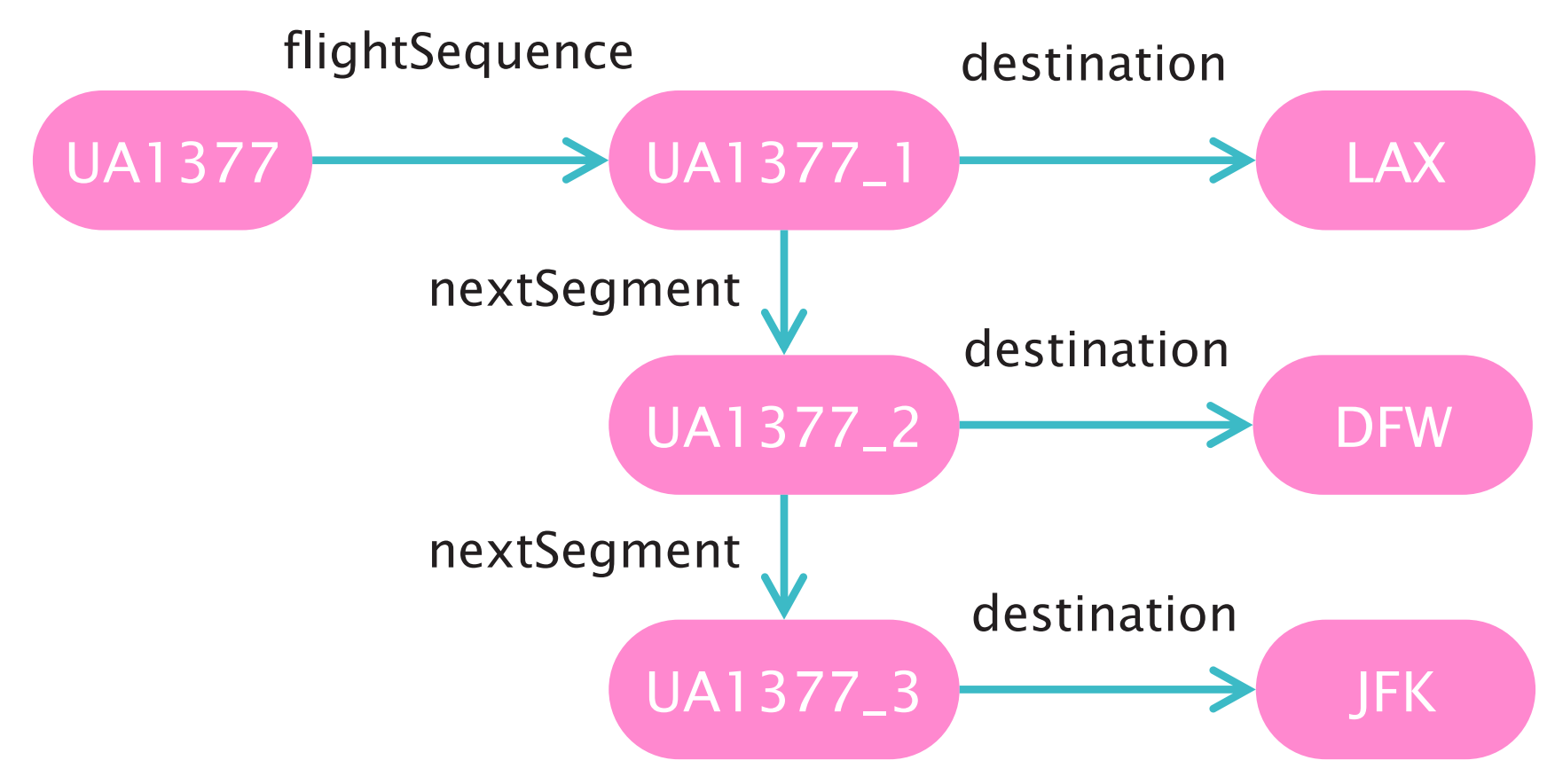




\section{Pattern 2: Sequence of arguments}

$\mathrm{T} \subseteq \forall$ flightSequence ${ }^{-}$. Flight

$T \subseteq \forall$ flightSequence. FlightSegment

$\mathrm{T} \subseteq \leq 1$ flightSequence

$\mathrm{T} \subseteq \forall$ nextSegment ${ }^{-}$. FlightSegment

$T \subseteq \forall$ nextSegment. FlightSegment

$T \subseteq \leq 1$ nextSegment

$\mathrm{T} \subseteq \forall$ destination $^{-}$. FlightSegment

$\mathrm{T} \subseteq \forall$ destination. Airport
(flightSequence rdfs:domain Flight)

(flightSequence rdfs:range FlightSegment)

(flightSequence is functional)

(nextSegment rdfs:domain FlightSegment)

(nextSegment rdfs:range FlightSegment)

(nextSegment is functional)

(destination rdfs:domain FlightSegment)

(destination rdfs:range Airport)

FlightSegment $\subseteq=1$ destination $\Pi \leq 1$ nextSegment

FinalFlightSegment $\equiv$ FlightSegment $\Pi=0$ nextSegment 
University of

(3) Southampton

\section{Value Partitions and Value Sets}




\section{Descriptive Features}

Descriptive features are quite common in ontologies:

- Size $=\{$ small, medium, large $\}$

- Risk = \{dangerous, risky, safe $\}$

- Health status = \{good health, medium health, poor health $\}$

Also called "qualities", "modifiers" and "attributes"

- A property can have only one value for each feature to ensure consistency

Three main approaches:

- Enumerated individuals (a value set)

- Disjoint classes (a value partition)

- Datatype values (not considered in this lecture) 


\section{Value Sets}

Values of descriptive feature are individuals

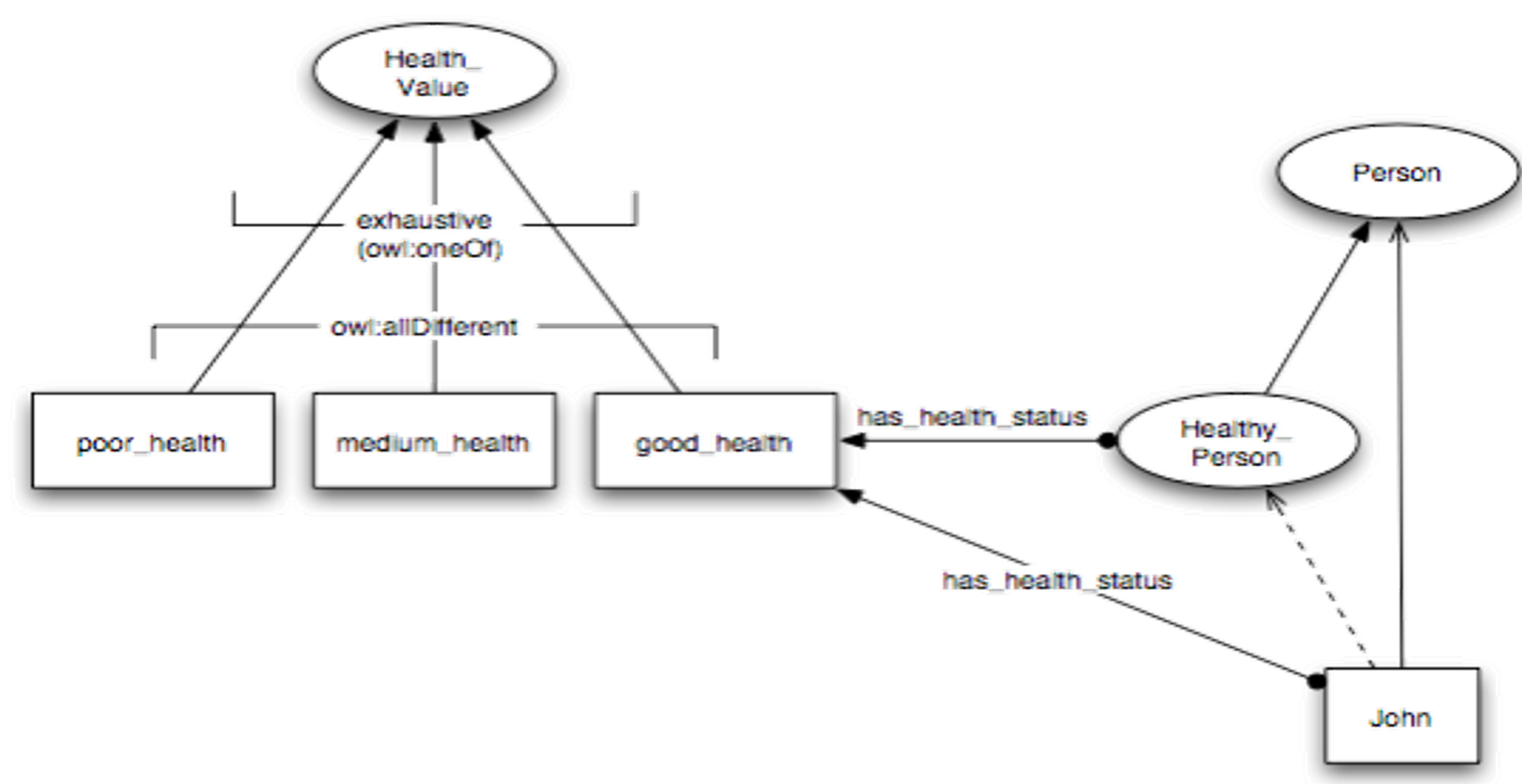




\section{Value Sets}

A health value can be either poor, medium or good:

HealthValue $\equiv\{$ poorHealth, mediumHealth, goodHealth $\}$

Poor, medium and good are all different from each other: poorHealth $\neq$ mediumHealth

poorHealth $\neq$ goodHealth

mediumHealth $\neq$ goodHealth

A healthy person is a person who has some health status which is the value good:

HealthyPerson $\equiv$ Person $\Pi \exists$ hasHealthStatus. \{ goodHealth \} 


\section{Notes on Value Sets}

Need axioms to set the three health values to be different from each other

- This way, a person cannot have more than one health value at a time

Values cannot be further partitioned

- e.g. cannot have fairly_good_health as a subtype of good_health

Only one set of values is allowed for a feature

- The class HealthValue cannot be equivalent to more than one set of distinct values

- Doing so will cause inconsistencies 


\section{Value Partitions}

Values of descriptive features are disjoint subclasses:

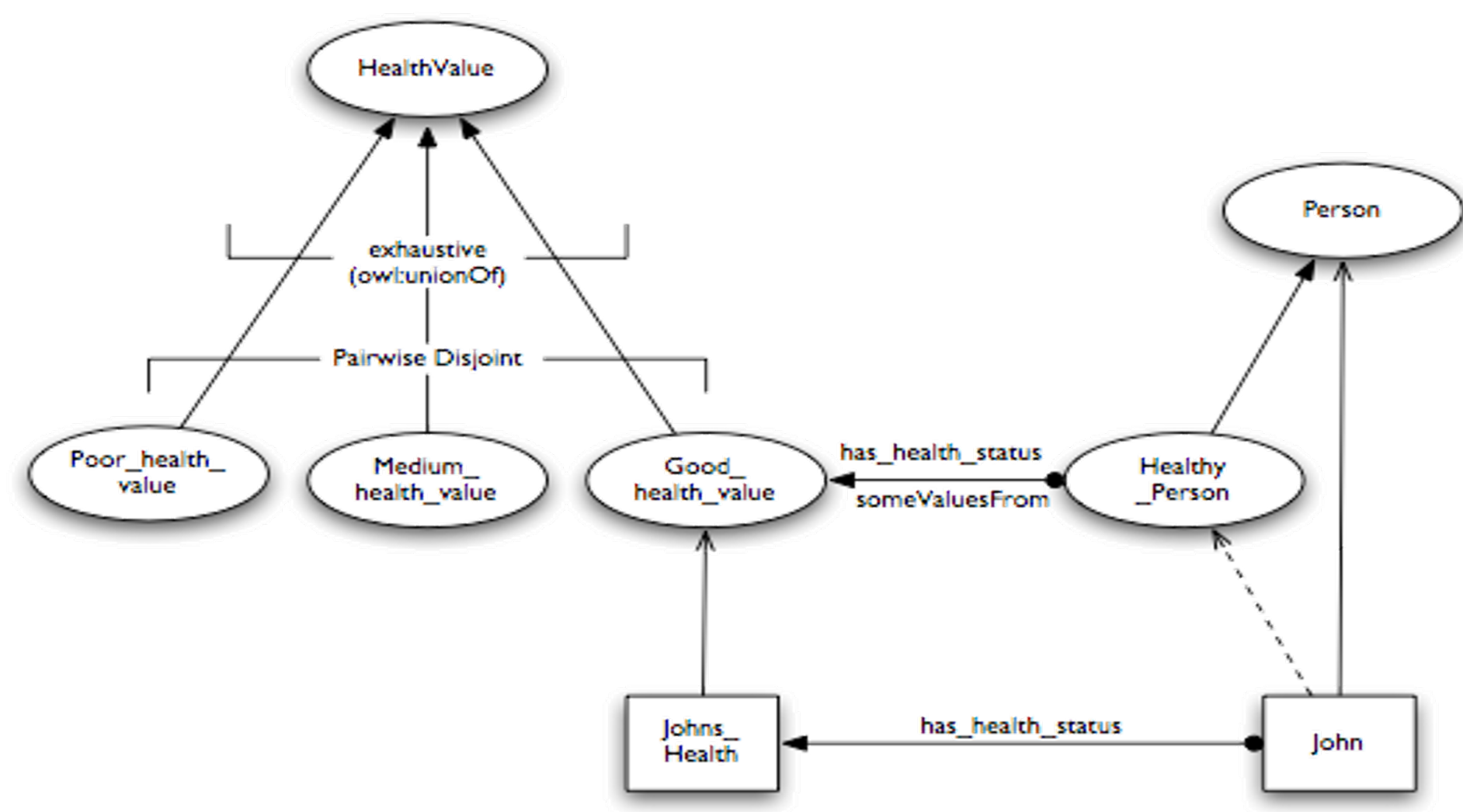




\section{Value Partitions}

Poor, medium and good are types of health value:

PoorHealth $\subseteq$ HealthValue

MediumHealth $\subseteq$ HealthValue

GoodHealth $\sqsubseteq$ HealthValue

Covering axiom (the only types of health value are poor, medium and good):

HealthValue $\equiv$ PoorHealth $\sqcup$ MediumHealth $\sqcup$ GoodHealth

Poor, medium and good are pairwise disjoint:

PoorHealth $\sqcap$ MediumHealth $\equiv \perp$

PoorHealth $\sqcap$ GoodHealth $\equiv \perp$

MediumHealth $\sqcap$ GoodHealth $\equiv \perp$

A healthy person is a person who has some health status which is an instance of good

HealthyPerson $\equiv$ Person $\Pi \exists$ hasHealthStatus. GoodHealth 


\section{Value Partitions}

The instance JohnsHealth can be made anonymous

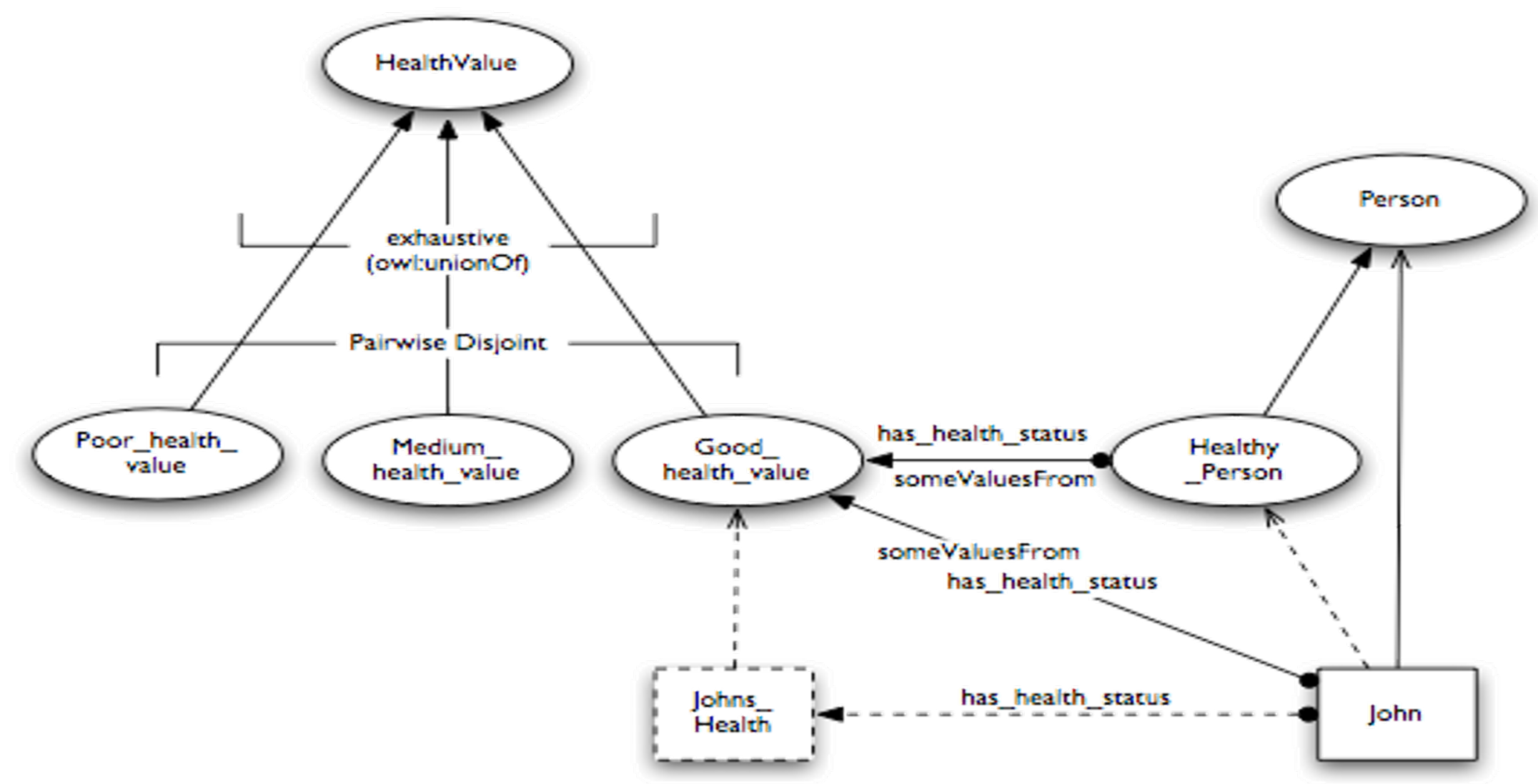




\section{Notes on Value Partitions}

Values can be further partitioned

- Simply add subclasses to the value classes

Can have alternative partitions of the same feature

OWL 2 contains specific support for defining disjoint unions

$$
\begin{gathered}
C \equiv C_{1} \sqcup C_{2} \sqcup \cdots \sqcup C_{n} \\
C_{1} \sqcap C_{2} \equiv \perp \\
C_{1} \sqcap C_{3} \equiv \perp \\
\cdots \\
C_{n-1} \sqcap C_{n} \equiv \perp
\end{gathered}
$$


University of

(3) Southampton

\section{Part-Whole Hierarchies}




\section{Meronymies (part-whole relations)}

Taxonomies are not the only hierarchical relation that we wish to model
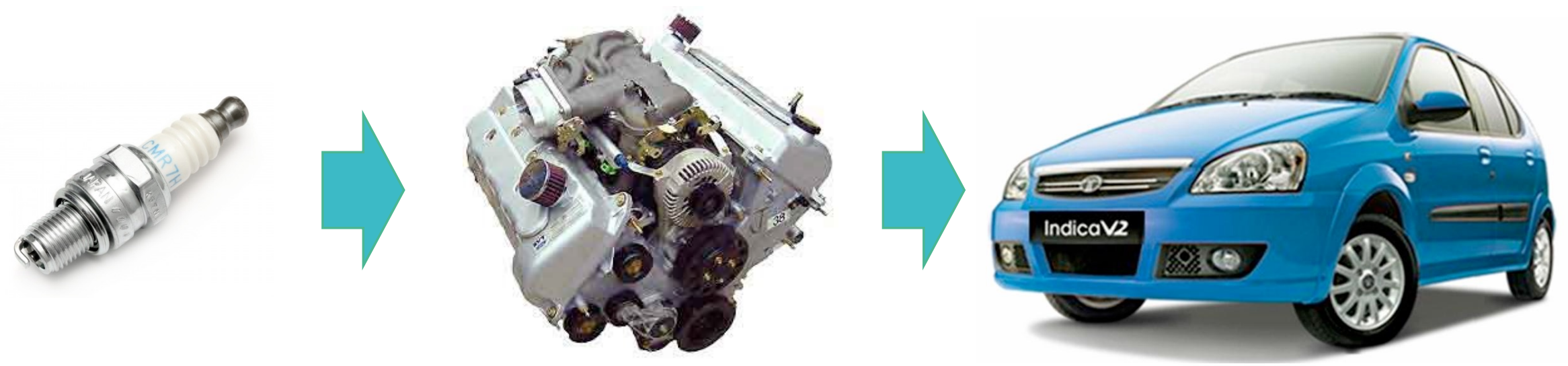

- A spark plug isn't a kind of engine (class-instance)

- A spark plug is a part of an engine 


\section{Simple Part-Whole Representation}

We need two properties:

- partOf (a transitive property)

- directPartOf (a subproperty of partOf)

$$
\begin{aligned}
& \text { part of } \circ \text { partOf } \subseteq \text { partOf } \\
& \text { directPartOf } \subseteq \text { partOf }
\end{aligned}
$$




\section{Part-Whole Hierarchies}

Represent part-whole relationships between classes using existential restrictions:

Every spark plug is a direct part of some engine: SparkPlug $\subseteq \exists$ directPartOf. Engine Every engine is a direct part of some car: Engine $\subseteq \exists$ directPartOf. Car

Every wheel is a direct part of some car: Wheel $\sqsubseteq \exists$ directPartOf. Car 


\section{Defining Classes of Parts}

Extend the ontology with classes of parts for each level, so that the reasoner can automatically derive a class hierarchy:

A car part is a part of some car:

CarPart $\equiv \exists$ partOf. Car

A direct car part is a direct part of some car:

DirectCarPart $\equiv \exists$ directPartOf. Car

An engine part is a part of some engine:

EnginePart $\equiv \exists$ partOf. Engine

A reasoner will infer that EnginePart $\subseteq$ CarPart (but not EnginePart $\subseteq$ DirectCarPart) 


\section{Fault Location}

Once we have a meronymy, we can use it to inherit features within that hierarchy

For example, a reasoner could infer that a fault in a part is a fault in a whole

- Need a new property for the location of a fault: hasLocus

- Need a new class for faults: Fault

We can then define general types of located faults:

FaultInCar $\equiv$ Fault $\sqcap$ ヨhasLocus. CarPart

FaultInEngine $\equiv$ Fault $\sqcap$ $\exists$ hasLocus. EnginePart 


\section{Fault Location}

Now we can define specific types of located fault:

$$
\begin{gathered}
\text { DirtySparkPlug } \subseteq \text { Fault } \sqcap \text { } \exists \text { hasLocus. SparkPlug } \\
\text { FlatTyre } \subseteq \text { Fault } \sqcap \text { } \exists \text { hasLocus. Wheel }
\end{gathered}
$$

The definition of the hierarchy allows a reasoner to infer that:

$$
\begin{gathered}
\text { DirtySparkPlug } \subseteq \text { FaultInCar } \\
\text { DirtySparkPlug } \subseteq \text { FaultInEngine } \\
\text { FlatTyre } \subseteq \text { FaultInCar }
\end{gathered}
$$

But not:

$$
\text { FlatTyre ㄷ FaultInEngine }
$$


University of

Southampton

Further Reading 


\section{SWBP Notes}

Defining $\mathrm{N}$-ary Relations on the Semantic Web http://www.w3.org/TR/swbp-n-aryRelations

Representing Specified Values in OWL http://www.w3.org/TR/swbp-specified-values

Simple part-whole relations in OWL Ontologies http://www.w3.org/2001/sw/BestPractices/OEP/SimplePartWhole/ 